Radiologe 2018 · 58:965-967

https://doi.org/10.1007/s00117-018-0447-8

(C) Springer Medizin Verlag GmbH, ein Teil von Springer Nature 2018

CrossMark

\title{
M.-A. Weber
}

Institut für Diagnostische und Interventionelle Radiologie, Universitätsmedizin Rostock, Rostock, Deutschland

\section{Bildgebende Diagnostik des Ellenbogens}

behandelnden als auch die diagnostizierenden Ärzte gestellt. Andererseits beträgt die Anzahl der dezidierten Ellenbogenuntersuchungen im Vergleich zur Anzahl der Untersuchungen anderer großer Gelenke selbst in muskuloskelettal fokussierten Einrichtungen im Schnitt bei deutlich unter $10 \%$ der Untersuchungen. Angesichts der Seltenheit der Untersuchungen an sich, kombiniert mit der komplexen Anatomie und den subtilen Befunden, möchte dieses Themenheft auf anschauliche und komprimierte Weise eine Fortbildung zu den verschiedenen Bereichen der bildgebenden Ellenbogengelenkdiagnostik vermitteln, von Frakturen über Bandverletzungen, Nervenschäden bis hin zu Normvarianten.

Das Themenheft startet mit einem Beitrag von Prof. Dr. Tischer zum Thema „Was erwartet der Orthopäde und Unfallchirurg vom Radiologen bei der Bildgebung des Ellenbogens?". Prof. Tischer betont vor allem die Wichtigkeit einer klaren und guten Kommunikation zwischen behandelndem und diagnostischem Arzt, insbesondere bei komplexen Fragestellungen.

Es folgt ein Beitrag von Prof. Dr. Gierer zu den typischen Frakturen und Luxationen am Ellenbogengelenk und deren Behandlung. Radiuskopffrakturen stellen mit einer Häufigkeit von $26 \%$ den größten Anteil der knöchernen Verletzungen am Ellenbogen dar, während die traumatische Luxation des Ellenbogengelenks mit einer Häufigkeit von $20 \%$ aller Verrenkungen nach der Schulterluxation die zweithäufigste Verrenkung großer Gelenke darstellt.

Es schließt sich ein Beitrag von Herrn Jäschke zu der Sonographie des El- lenbogengelenks an, in dem die Standardschnitte und die Normalanatomie dargestellt werden. Der Artikel arbeitet die Empfehlungen der DEGUM und der ESSR für die Sonographie des Ellenbogengelenkes ein. Die Sonographie ermöglicht eine dynamische Untersuchung und bietet die Möglichkeit zur gleichzeitigen Beurteilung des kontralateralen Ellenbogens, der als Referenz genutzt werden kann.

$P D$ Dr. Fischer führt in seinem Beitrag zur Ultraschalldiagnostik muskuloligamentärer Verletzungen sowie Insertionstendopathien am Ellenbogengelenk sonopathologische Befunde der häufigsten Entitäten vor. Da gerade in der posttraumatischen Akutphase die klinische Untersuchung oft nur schwer durchführbar ist, empfiehlt PD Dr. Fischer in der Akutdiagnostik den kostengünstigen sowie schnell verfügbaren Ultraschall als wichtige Bildgebungsmodalität.

Es folgt ein Beitrag von Dr. Janßen zu den Band- und Sehnenverletzungen des Ellenbogens in der Magnetresonanztomographie. Er führt aus, dass insbesondere die Kenntnis von Varianten einzelner Bänder, die in seinem Beitrag beschrieben werden, wie z.B. das partiell streifige Erscheinungsbild des ulnaren Kollateralbandes und des lateralen, ulnaren Kollateralbandes, in der Differenzierung von Zerrung und Partialrupturen sehr hilfreich ist. Spezielle Schichtführungen zur Beurteilung von Sehnen und Bändern werden ebenfalls beschrieben und den MRT-Befunden sehr illustrative, graphische Darstellungen gegenübergestellt.

Es folgt ein Beitrag zu den Entrapment- und Non-Entrapment-Syndromen am Ellenbogen von Dr. Godel. Er 
führt schön aus, dass mithilfe der bildgebenden Nervendarstellung in einem Untersuchungsgang die nervalen Strukturen am Ellenbogengelenk komplett erfasst und fokale von multifokalen Nervenerkrankungen differenziert werden können.

Das Themenheft wird mit einem reichlich illustrierten Beitrag zu Normvarianten und Pitfalls rund um den Ellenbogen von Dr. Müller geschlossen. Sehr illustrativ werden häufigere, aber auch seltenere Normvarianten des Ellenbogengelenks beschrieben und einer ähnlich anmutenden Pathologie gegenübergestellt sowie klassische Fallstricke aufgezeigt.

Insgesamt hoffe ich, dass Ihnen, liebe Leserinnen und Leser, dieses Themenheft einen umfassenden Überblick über die aktuellen diagnostischen Herausforderungen bei diesem speziellen Thema der Radiologie des Ellenbogens liefert. Ich wünsche Ihnen sehr viel Freude beim Lesen und hoffe, Sie finden diese Zusammenstellung interessant und hilfreich für Ihre praktische Tätigkeit.

Mit herzlichen Grüßen,

Ihr

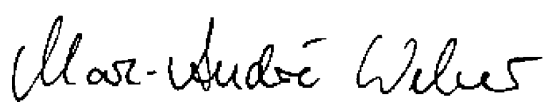

Prof. Dr. Marc-André Weber, M.Sc.

\section{Korrespondenzadresse}

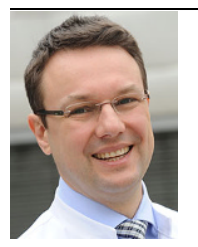

Prof. Dr. M.-A. Weber, M.Sc. Institut für Diagnostische und Interventionelle Radiologie, Universitätsmedizin Rostock Ernst-Heydemann-Str. 6, 18057 Rostock, Deutschland marc-andre.weber@ med.uni-rostock.de

Interessenkonflikt. M.-A. Weber gibt an, dass kein Interessenkonflikt besteht.

Laver, L., Landreau, P., Seil, R., Popovic, N. (Eds.)

\section{Handball Sports Medicine}

Basic Science, Injury Management and Return to Sport

Berlin Heidelberg: Springer-Verlag 2018, 1. Auflage, XXVII, 653 S., 254 Abb., (ISBN: 978-3-662-55891-1), Hardcover: 228,98 EUR, eBook: 178,49 EUR

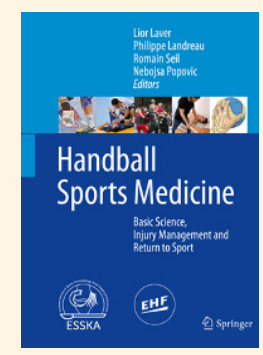

Ein sehr spannendes Thema für den sportmedizinisch interessierten Mediziner, der sich mit den spezifischen Aspekten dieser faszinierenden Sportart auseinander setzen möchte. Zeitgemäß wird ein Online-Zugang bzw. eine eBook-Version bereitgestellt.

Sowohl die Teilabschnitte als auch die Anordnung der Kapitel sind logisch und didaktisch sinnvoll aufeinander aufgebaut. Im ersten Teil wird die Sportart Handball allumfassend vorgestellt. Beginnend mit den physischen Voraussetzungen der Sportler/innen bis zum eindrücklichen Kapitel 4 "The Shoulder Profile in Team Handball" und den biomechanischen Überlegungen in den Kapiteln 5 \& 6 der unteren bzw. oberen Extremität. Diese Kapitel sind zum Verständnis der sportarttypischen Verletzungsmuster didaktisch sehr wertvoll. Der zweite Abschnitt befasst sich mit der Rolle des Sportmediziners im handballspezifischen Umfeld. Dieser Abschnitt ist äußerst relevant und elementar, um die Rolle des Mediziners in diesem Betätigungsfeld nachzuvollziehen.

Der dritte Abschnitt stellt die Verletzungsmuster nach Körperregion von Kopf bis Fuß dar. In diesen Kapiteln werden die Pathologien systematisch prägnant aufgearbeitet und vor allem sportartspezifische Aspekte berücksichtigt, die Konsequenzen für die Therapie haben.

Der vierte Abschnitt befasst sich mit den sehr wichtigen Themen Prävention, Rehabilitation und physische Vorbereitung. Hervorheben muss man die Kapitel 30 "Implementing Handball Injury Prevention Exercise Programs: A Practical Guideline" und 34 "A Biomechanical Perspective on Rehabilitation of ACL Injuries in Handball". Das Kapitel 30 stellt eindrücklich und sehr praxisorientiert Übungen mit einprägsamen Abbildungen für den/die Handballer/in vor. Das Kapitel 34 vermittelt sehr zielgerichtet Hintergrundwissen für die sehr schwerwiegenden Kreuzbandverletzungen, das für die Rehabilitation und Prävention äußerst wichtig ist. Abgerundet wird dieses Kapitel durch didaktisch einprägsame und farblich gekennzeichnete Textfelder.

Der vorletzte und letzte Abschnitt runden die Thematik des Buchs mit Abhandlungen über spezifische Gesichtspunkte im Jugendbzw. Frauenhandball, über Doping und über psychologische Aspekte umfänglich ab.

Im Fachbereich Sportmedizin ist den Herausgebern eine tiefgreifende Monographie über die Sportart Handball gelungen. Dieses Buch eröffnet auch dem nicht im "Handballsport aufgewachsenen" Mediziner sportmedizinisch und sportartspezifisch relevantes Wissen auf einprägsame Weise. Dieses Werk sollte für den im Handballsport engagierten Mediziner eine unverzichtbare Lektüre sein.

\section{Spies (Bad Rappenau)}


Hier steht eine Anzeige.

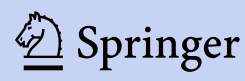

